\title{
Strong Geodetic Number in Some Networks
}

\author{
Huifen $\mathrm{Ge}^{1}$, Zhao Wang ${ }^{2}$ \& Jinyu Zou ${ }^{3}$ \\ ${ }^{1}$ School of Mathematics and Statistics, Qinghai Normal University, Xining, Qinghai, China \\ ${ }^{2}$ College of Science, China Jiliang University, HangZhou, China \\ ${ }^{3}$ School of Computer Sciences, Qinghai Normal University, Xining, Qinghai, China \\ Correspondence: Zhao Wang, College of Science, China Jiliang University, HangZhou, China. \\ E-mail: wangzhao@cjlu.edu.cn
}

Received: January 13, 2019 Accepted: February 11, 2019 Online Published: February 20, 2019

doi:10.5539/jmr.v11n2p20 URL: https://doi.org/10.5539/jmr.v11n2p20

\begin{abstract}
A vertex subset $S$ of a graph is called a strong geodetic set if there exists a choice of exactly one geodesic for each pair of vertices of $S$ in such a way that these $\left(\begin{array}{c}|S| \\ 2\end{array}\right)$ geodesics cover all the vertices of graph $G$. The strong geodetic number of $G$, denoted by $\operatorname{sg}(G)$, is the smallest cardinality of a strong geodetic set. In this paper, we give an upper bound of strong geodetic number of the Cartesian product graphs and study this parameter for some Cartesian product networks.
\end{abstract}

Keywords: strong geodetic number, cartesian product, hyper Peterson network, torus network, cube

\section{Introduction}

All graphs considered in this paper are connected, simple, undirected and finite. We refer to the book (Bondy \& Murty, 2008) for graph theoretical notation and terminology not described here. The distance between vertices $u$ and $v$ of a graph $G$ denoted by $d_{G}(u, v)$, is the length of a shortest path between $u$ and $v$, a $u$-v geodesic is a shortest $u-v$ path of $G$. The diameter of a graph $G \operatorname{diam}(G)$ is the maximum distance between any two vertices. We use the notation $[n]=\{1,2, \ldots, n\}$, $V\left(P_{n}\right)=[n]$ for any $n \geq 2$ as well as $V\left(C_{n}\right)=[n]$ for any $n \geq 3$, where the edges of $P_{n}$ and $C_{n}$ are defined in the natural way.

The problem of covering a graph by geodesic is a topic researched widely in graph theory, application to channel design of aircraft and vessels, social transport networks and so on. The geodetic set is a vertex subset $S$, such that each vertex of $G$ lies on some shortest paths between a pair of vertices from $S$. (Harary et al., 1993) introduced the geodetic problem, is to find a geodetic set of minimum size. A class of covering problem is formed by path covering that include the edge covering problem, the geodesic covering problem, the induced path covering problem and path covering problem. The parameters are used in analysis of structural behavior of product networks by (Buckley et al., 1998; Chartrand et al., 2000 $\& 2002)$.

Let $G=(V, E)$ be a graph. Given a set $S \subseteq V$, for each pair of vertices $\{x, y\} \subseteq S, x \neq y$, let $\widetilde{P}(x, y)$ be a selected fixed shortest path between $x$ and $y$. Then we set

$$
\widetilde{I}(S)=\{\widetilde{P}(x, y): x, y \in S\}
$$

and let $V(\widetilde{I}(S))=\cup_{\widetilde{P} \in \widetilde{I}(S)} V(\widetilde{P})$. If $V(\widetilde{I}(S))=V(G)$ for some $V(\widetilde{I}(S))$, then the set $S$ is called a strong geodetic set. The strong geodetic problem is to find a minimum strong geodetic set $S$ of $G$, the minimum cardinality of a strong geodetic set is defined as the strong geodetic number, denoted by $\operatorname{sg}(G)$.

Later, this concept and other related invariants in various classes of graph are considered in several literature (Atici, 2002; Brešar et al., 2011; Brešar \& Tepeh, 2008; Chartrand et al., 2002; Fitzpatrick, 1999; Manuel et al., 2017; Ye et al., 2007). More latest papers see(Iršičc, 2018; Klavžar \& Manuel, 2018; Manuel et al., 2018).

Recently there has been an increasing interest in a class of interconnection networks called Cartesian product networks; see (Bao et al., 1998; Day \& AL-Ayyoub, 1997; Ku et al., 2003). The Cartesian product of two graphs $G$ and $H$, denoted by $G \square H$, is a graph with vertex set $V(G) \times V(H)$ such that $(u, v)$ and $\left(u^{\prime}, v^{\prime}\right)$ are adjacent if and only if either $u=u^{\prime}$ and $v v^{\prime} \in E(H)$, or $v=v^{\prime}$ and $u u^{\prime} \in E(G)$. Note that this product is commutative, that is $G \square H=H \square G$. For a vertex $v \in V(H)$, set $G^{v}=\{(u, v) \in V(G \square H) \mid u \in V(G)\}, G^{v}$ is called a $G$-layer of $G \square H$. We consider $G^{v}$ as the corresponding induced subgraph, then $G^{v}$ is isomorphic to $G$. For a vertex $u \in V(H)$, the $H$-layer is defined as $H^{u}=\{(u, v) \in V(G \square H) \mid v \in V(H)\}$, it is isomorphic to $H$.

Klavžar and Manuel (Klavžar \& Manuel, 2018) have given a general upper bound on the Cartesian product of a path with 
an arbitrary graph and showed that the bound is tight on flat grids and flat cylinders. In this paper, we investigate the strong geodesic problem on the torus network and some other Cartesian product networks. In Section 2, we introduce the notation and basic concepts. In Section 3, we obtain a lower bound of $s g(G)$ and an upper bound of $s g(G \square H)$, and we obtain the exact value of the strong geodetic number of the two hyper Peterson graph $\mathrm{HP}_{3}$ and $\mathrm{HP}_{4}$. Then, we present an upper bound of $\operatorname{sg}\left(C_{r} \square C_{n}\right)$. Finally, we study the exact value of $\operatorname{sg}\left(Q_{n}\right)$ for $n \leq 5$ and an upper bound when $n \geq 6$.

\section{Main Results}

The following result is well-known.

Lemma 1 (Hammack et al.,2001) Let $(u, v)$ and $\left(u^{\prime}, v^{\prime}\right)$ be arbitrary vertices of Cartesian product $G \square H$. Then

$$
d_{G \square H}\left((u, v),\left(u^{\prime}, v^{\prime}\right)\right)=d_{G}\left(u, u^{\prime}\right)+d_{H}\left(v, v^{\prime}\right) .
$$

By Lemma 1, the diameter of the Cartesian product is equal to the sum of the diameter of the original two graphs, that is, $\operatorname{diam}(G \square H)=\operatorname{diam}(G)+\operatorname{diam}(H)$.

For some special graphs, we have following results.

Observation 1 (1) For a complete graph $K_{n}, s g\left(K_{n}\right)=n$.

(2) For a tree $T$ with $p$ pendant vertices, $s g(T)=p$.

(3) For a cycle $C_{n}, \operatorname{sg}\left(C_{n}\right)=3$.

(4) For a wheel graph $W_{1, n}$,

$$
\operatorname{sg}\left(W_{1, n}\right)= \begin{cases}4, & \text { if } 3 \leq n \leq 6 \\ \left\lceil\frac{n}{2}\right\rceil, & \text { if } n \geq 7\end{cases}
$$

\subsection{Bounds for $\operatorname{sg}(G)$ and $\operatorname{sg}(G \square H)$}

First, we give two general results for strong geodetic number.

Lemma 2 For a connected graph $G$, we have

$$
s g(G) \geq\left\lceil\frac{d-3+\sqrt{(d-3)^{2}+8|V(G)|(d-1)}}{2(d-1)}\right\rceil,
$$

where $d$ is the diameter of graph $G$.

Proof. From the definition of the strong geodetic set $S$, for each pair of vertices $\{x, y\} \subseteq S$, there are at most $\operatorname{diam}(G)-1$ internal vertices are covered by $x-y$ shortest path, that is

$$
V|\widetilde{P}(x, y)|-\{x, y\} \leq \operatorname{diam}(G)-1 .
$$

The collection $\widetilde{I}(S)$ of geodesics consist of exactly $\left(\begin{array}{c}s g(G) \\ 2\end{array}\right)$ paths, then

$$
s g(G)+\left(\begin{array}{c}
s g(G) \\
2
\end{array}\right)(\operatorname{diam}(G)-1) \geq|V(G)| .
$$

as desired.

Next, we give an upper bound of $s g(G \square H)$.

Theorem 1 Let $G$ and $H$ be two graphs of order $n$ and $m$, respectively. Then

$$
s g(G \square H) \leq \min \{n(\operatorname{sg}(H)-1)+1, m(\operatorname{sg}(G)-1)+1\} .
$$

Proof. Let $s g(G)=p$ and $s g(H)=q$, and let $V(G \square H)=\left\{\left(u_{i}, v_{j}\right) \mid u_{i} \in V(G), v_{j} \in V(H)\right\}(i=1,2, \cdots, n ; j=1,2, \cdots, m)$. Then there are $n$ H-layers $H^{u_{1}}, H^{u_{2}}, \cdots, H^{u_{n}}$ in $G \square H$. Denote by $S=\left\{v_{j_{1}}, v_{j_{2}}, \cdots, v_{j_{q}}\right\}\left(j_{1}, j_{2}, \cdots, j_{q} \in[m]\right)$ the geodetic set of $H$. Then we let $S_{i} \subseteq\left\{\left(u_{i}, v_{j_{1}}\right),\left(u_{i}, v_{j_{2}}\right), \cdots,\left(u_{i}, v_{j_{q}}\right)\right\}$ be the geodetic set in $H^{u_{i}}$ corresponding to $S$ in $H$, where $1 \leq i \leq n$. Choose

$$
S_{1}=\left\{\left(u_{1}, v_{j_{1}}\right),\left(u_{1}, v_{j_{2}}\right), \cdots,\left(u_{1}, v_{j_{q}}\right)\right\},
$$




$$
\begin{gathered}
S_{2}=\left\{\left(u_{2}, v_{j_{1}}\right),\left(u_{2}, v_{j_{2}}\right), \cdots,\left(u_{2}, v_{j_{q-1}}\right)\right\}, \\
\vdots \\
S_{n}=\left\{\left(u_{n}, v_{j_{1}}\right),\left(u_{n}, v_{j_{2}}\right), \cdots,\left(u_{n}, v_{j_{q-1}}\right)\right\} .
\end{gathered}
$$

Then $V\left(\widetilde{I}\left(\cup_{i=1}^{n} S_{i}\right)\right)=\cup_{i=1}^{n} V\left(H^{u_{i}}\right)=V(G \square H)$.

Clearly, $V\left(\widetilde{I}\left(S_{1}\right)\right)=V\left(H^{u_{1}}\right)$, the geodesic between one vertex of $S_{2}$ and $\left(u_{1}, v_{j_{q}}\right)$ can cover the vertex $\left(u_{2}, v_{j_{q}}\right)$, and so $V\left(H^{u_{2}}\right) \subset V\left(\widetilde{I}\left(S_{2} \cup\left(u_{1}, v_{j_{q}}\right)\right)\right)$. Furthermore, we have $V\left(\widetilde{I}\left(S_{1} \cup S_{2}\right)\right)=V\left(H^{u_{1}}\right) \cup V\left(H^{u_{2}}\right)$. Similarly, we have $V\left(\widetilde{I}\left(S_{1} \cup S_{3}\right)\right)=$ $V\left(H^{u_{1}}\right) \cup V\left(H^{u_{3}}\right), \cdots, V\left(\widetilde{I}\left(S_{1} \cup S_{n}\right)\right)=V\left(H^{u_{1}}\right) \cup V\left(H^{u_{n}}\right)$. Since $V\left(\widetilde{I}\left(\cup_{i=1}^{n} S_{i}\right)\right)=\cup_{i=1}^{n} V\left(H^{u_{i}}\right)=V(G \square H)$, it follows that $s g(G \square H) \leq n(q-1)+1$.

By the symmetry, we have $s g(G \square H) \leq m(p-1)+1$, and hence $s g(G \square H) \leq \min \{n(q-1)+1, m(p-1)+1\}$.

\subsection{Hyper Peterson Network}

An $n$-dimensional hyper Peterson network $H P_{n}$ is the product of the well-known Peterson graph and $Q_{n-3}$ (Das et al., 1995), where $n \geq 3$ and $Q_{n-3}$ denotes an (n-3)-dimensional hypercube. The case $n=3$ and 4 of hyper Peterson networks are depicted in Figure 1. Note that $H P_{3}$ is just the Peterson graph.

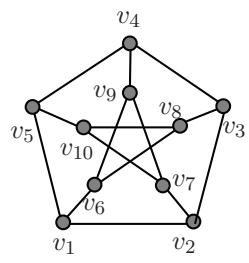

(a)

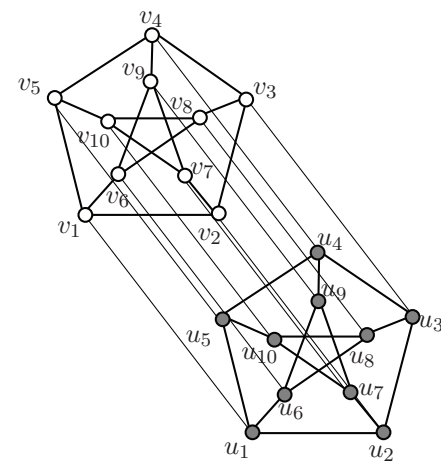

$(b)$

Figure 1. (a) Peterson graph, (b) The network $\mathrm{HP}_{4}$

For a Peterson graph, we have the following result.

Theorem 2 For a Peterson graph $\mathrm{HP}_{3}, \operatorname{sg}\left(\mathrm{HP}_{3}\right)=4$.

Proof. From Lemma 2, we have $\operatorname{sg}\left(H P_{3}\right) \geq 4$. Let $S=\left\{v_{1}, v_{3}, v_{9}, v_{10}\right\}$ and $\widetilde{I}(S)=\left\{v_{1}-v_{2}-v_{3}, v_{1}-v_{6}-v_{9}, v_{1}-v_{5}-v_{10}, v_{3}-v_{4}-v_{9}\right.$, $\left.v_{3}-v_{8}-v_{10}, v_{9}-v_{7}-v_{10}\right\}$. Then $V(\widetilde{I}(S))=V\left(H P_{3}\right)$, and hence $\operatorname{sg}\left(H P_{3}\right)=4$.

Above example shows equality holding in Lemma 2.

Theorem 3 For a network $\mathrm{HP}_{4}, \operatorname{sg}\left(H P_{4}\right)=6$.

Proof. For the network $H P_{4}$, there are two copies of Peterson graphs, say $H P_{3}$ and $H P_{3}^{\prime}$. Let $V\left(H P_{3}\right)=\left\{v_{i} \mid 1 \leq i \leq 10\right\}$ and $V\left(H P_{3}^{\prime}\right)=\left\{u_{j} \mid 1 \leq j \leq 10\right\}$.

We first prove $5 \leq s g\left(H P_{4}\right) \leq 6$. Since $\operatorname{diam}\left(H P_{4}\right)=2$ and $\left|V\left(H P_{4}\right)\right|=20$, it follows from Lemma 2 that $s g\left(H P_{4}\right) \geq 5$. Choose $S=\left\{v_{1}, v_{7}, v_{9}, u_{3}, u_{4}, u_{8}\right\}$. Let $\widetilde{I}(S)=\left\{v_{1}-v_{2}-v_{7}, v_{1}-v_{6}-v_{9}, v_{1}-v_{2}-v_{3}-u_{3}, v_{1}-v_{5}-u_{5}-u_{4}, v_{1}-u_{1}-u_{6}-u_{8}, v_{7}-v_{2}-u_{2}-u_{3}, v_{7}-u_{7}-\right.$ $\left.u_{9}-u_{4}, v_{7}-v_{10}-u_{10}-u_{8}, v_{9}-v_{6}-v_{8}-u_{8}, v_{9}-v_{4}-u_{4}\right\}$. Then $V(\widetilde{I}(S))=V\left(H P_{4}\right)$, and hence $\operatorname{sg}\left(H P_{4}\right) \leq 6$.

We claim that $s g\left(H P_{4}\right)=6$. Assume, to the contrary, that $\operatorname{sg}\left(H P_{4}\right)=5$. Clearly, there are 10 geodesics. If $\left|S \cap V\left(H P_{3}\right)\right|=$ 4 and $\left|S \cap V\left(H P_{3}^{\prime}\right)\right|=1$, then the 6 geodesics constructed by $\left\{v_{1}, v_{2}, \cdots, v_{10}\right\}$ can cover at most 10 vertices of $H P_{3}$, and other 4 geodesics between one vertex of $\left\{v_{1}, v_{2}, \cdots, v_{10}\right\}$ and one vertex of $\left\{u_{1}, u_{2}, \cdots, u_{10}\right\}$ can cover at most 8 vertices in $H P_{3}^{\prime}$. So $V(\widetilde{I}(S)) \leq V\left(H P_{4}\right)$, a contradiction. 
Suppose $\left|S \cap V\left(H P_{3}\right)\right|=3$ and $\left|S \cap V\left(H P_{3}^{\prime}\right)\right|=2$. Since the distance of any two vertices of $\left\{v_{1}, v_{2}, \cdots, v_{10}\right\}$ or $\left\{u_{1}, u_{2}, \cdots, u_{10}\right\}$ is at most 2 , and the distance between one vertex of $\left\{v_{1}, v_{2}, \cdots, v_{10}\right\}$ and one vertex of $\left\{u_{1}, u_{2}, \cdots, u_{10}\right\}$ is at most 3 , it follows that there are at most 6 pairs of vertices of $S$ with distance 3 and each geodesic with distance 3 covers at most 2 vertices outside of $S$. We claim that there are at least 5 geodesics with distance 3 . Assume, to the contrary, that there are 4 geodesics with distance 3. Since each geodesic with distance 3 covers at most 2 vertices outside of $S$ and each remaining geodesic covers at most one vertex outside of $S$, it follows that all the 10 geodesics cover at most 14 vertices outside of $S$ and 19 vertices of $H_{4}$, a contradiction. We distinguish the following cases to show this theorem by the value of $r$, where $r$ is the number of geodesics with distance 3 .

Case 1. $r=5$

If there are 5 geodesics with distance 2 , then the 10 geodesics exactly cover all vertices of $H P_{4}$. Without loss of generality, suppose $S \cap V\left(H P_{3}^{\prime}\right)=\left\{u_{1}, u_{3}\right\}, S \cap V\left(H P_{3}\right)=\left\{v_{9}, v_{10}, v_{i}\right\}$, where the vertex $v_{i}$ satisfies $d\left(v_{9}, v_{i}\right)=2$ and $d\left(v_{10}, v_{i}\right)=2$. Then $v_{i} \in\left\{v_{1}, v_{2}, v_{3}\right\}$. If $v_{i}=v_{1}$, then $d\left(u_{1}, v_{1}\right)=1$, a contradiction. If $v_{i}=v_{3}$, then $d\left(u_{3}, v_{3}\right)=1$, a contradiction. If $v_{i}=v_{2}$, then $d\left(u_{1}, v_{2}\right)=2$ and $d\left(u_{3}, v_{2}\right)=2$, also a contradiction.

Case 2. $r=6$

If there are 2 geodesics with distance 2 and every geodesic with distance 2 cover at most one vertex outside of $S$, the remaining 2 geodesics with distance 1 , then the 10 geodesics cover at most 14 vertices outside of $S$ and 19 vertices of $\mathrm{HP}_{4}$, and hence there are at most 3 geodesics with distance 2 .

Suppose there are 3 geodesics with distance 2 and one geodesic with distance 1 . For any vertex $v_{i} \in S \cap V\left(H P_{3}\right), u_{j} \in S \cap$ $V\left(H P_{3}^{\prime}\right)$, we have $d\left(v_{i}, u_{j}\right)=3$. Without loss of generality, let $S \cap V\left(H P_{3}^{\prime}\right)=\left\{u_{1}, u_{2}\right\}$. Clearly, $S \cap V\left(H P_{3}\right) \subset\left\{v_{4}, v_{8}, v_{9}, v_{10}\right\}$. If $S=\left\{u_{1}, u_{2}, v_{4}, v_{8}, v_{9}\right\}$, then $d\left(v_{8}, v_{9}\right)=1$. If $S=\left\{u_{1}, u_{2}, v_{4}, v_{8}, v_{10}\right\}$, then $d\left(v_{8}, v_{10}\right)=1$. If $S=\left\{u_{1}, u_{2}, v_{4}, v_{9}, v_{10}\right\}$, then $d\left(v_{4}, v_{9}\right)=1$. If $S=\left\{u_{1}, u_{2}, v_{8}, v_{9}, v_{10}\right\}, d\left(v_{8}, v_{10}\right)=1$, a contradiction.

Suppose there are 4 geodesics with distance 2. Without loss of generality, let $S \cap V\left(H P_{3}^{\prime}\right)=\left\{u_{1}, u_{3}\right\}$, and hence $S \cap$ $V\left(H P_{3}\right)=\left\{v_{7}, v_{9}, v_{10}\right\}$. Now $S=\left\{v_{7}, v_{9}, v_{10}, u_{1}, u_{3}\right\}$, then $d\left(v_{7}, v_{9}\right)=d\left(v_{7}, v_{10}\right)=1$, a contradiction.

\subsection{Torus Network}

In this section, we give an upper bound on the strong geodetic number of torus graphs. The strong geodetic number of the grids (product of two paths) and cylinders (product of a path and a cycle) were given in (Klavžar \& Manuel, 2018).

Theorem 4 (Klavzar \& Manuel, 2018) (1) If $2 \leq n \leq r$, then $\operatorname{sg}\left(P_{r} \square P_{n}\right) \leq\lceil 2 \sqrt{n}\rceil$.

(2) If $2 \leq n \leq r$, then $\operatorname{sg}\left(P_{r} \square C_{n}\right) \leq\lceil 2 \sqrt{n}\rceil$.

Theorem 5 If $3 \leq n \leq r$, then $\operatorname{sg}\left(C_{r} \square C_{n}\right) \leq\lceil 3 \sqrt{n}\rceil$.

Proof. In order to prove the upper bound, we only need to find a strong geodetic set of cardinality $\lceil 3 \sqrt{n}\rceil$. For convenience we use previous notation $V\left(C_{r}\right)=[r]=\{1,2, \ldots, r\}, V\left(C_{n}\right)=[n]=\{1,2, \ldots, n\}$. Let $a_{i}, b_{i}$ and $c_{i}$ are some vertices of the graph $C_{r} \square C_{n}$ for $i \in[k]$ and with

$$
\begin{aligned}
a_{i} & =(1,(i-1) k+1), \\
b_{i} & =\left(\left[\frac{r}{2}\right],(i-1) k+1\right), \\
c_{i} & =(r,(i-1) k+1) .
\end{aligned}
$$

Next we consider the following cases.

Case $1 n=k^{2}$.

Let $S=\left\{a_{1}, a_{2}, \ldots, a_{k}\right\} \cup\left\{b_{1}, b_{2}, \ldots, b_{k}\right\} \cup\left\{c_{1}, c_{2}, \ldots, c_{k}\right\}$ and $|S|=3 k=3 \sqrt{n}$. Next, we prove $V(\widetilde{I}(S))=V\left(C_{r} \square C_{n}\right)$ for some $\widetilde{I}(S)$. There are $r$ layers $C_{n}, n$ layers $C_{r}$, denoted by $C_{n}^{1}, C_{n}^{2}, \ldots, C_{n}^{r}$ and $C_{r}^{1}, C_{r}^{2}, \ldots, C_{r}^{n}$ respectively. We can see that the subgraph induced by

$$
V\left(C_{n}^{1}\right) \cup V\left(C_{n}^{2}\right) \cup \ldots \cup V\left(C_{n}^{\left\lceil\frac{r}{2}\right\rceil}\right)
$$

is isomorphic to $P_{\left\lceil\frac{r}{2}\right\rceil} \square C_{n}$. In order to show the set $S_{1}=\left\{a_{1}, a_{2}, \ldots, a_{k}\right\} \cup\left\{b_{1}, b_{2}, \ldots, b_{k}\right\}$ is a geodesic set of $P_{\left\lceil\frac{r}{2}\right\rceil} \square C_{n}$, it suffices to prove $V\left(\widetilde{I}\left(S_{1}\right)\right)=V\left(P_{\left\lceil\frac{r}{2}\right\rceil} \square C_{n}\right)$.

As $a_{i}$ and $a_{j}$ are both on the $C_{n}^{1}$-layer, the shortest path from $a_{i}$ to $a_{j}$ is unique. Then the geodesic $a_{i}-a_{j}$ is unique. $b_{i}$ and $b_{j}$ are on the $C_{n}^{\left[\frac{r}{2}\right]}$-layer, $a_{i}$ and $b_{i}$ are on the $C_{r}^{i}$-layer, so the geodesics $b_{i}-b_{j}$ and $a_{i}-b_{i}$ are unique. We select a fixed $a_{i}-b_{j}$ geodesic $(i \neq j)$ by the following way. 
Start from $a_{1}$, through vertex $(1,2)$ of $C_{n}^{1}$-layer, traverse the $P_{\left\lceil\frac{r}{2}\right\rceil}^{2}$-layer until vertex $\left(\left\lceil\frac{r}{2}\right\rceil, 2\right)$, through $C_{n}^{\left[\frac{r}{2}\right\rceil+1}$-layer to $b_{2}$, that is,

$$
a_{1}-(1,2)-\left(\left[\frac{r}{2}\right\rceil, 2\right)-b_{2}
$$

Then

$$
\begin{gathered}
a_{1}-(1,3)-\left(\left\lceil\frac{r}{2}\right\rceil, 3\right)-b_{3}, \\
\vdots \\
a_{1}-\left(1,\left\lfloor\frac{k}{2}\right\rfloor+1\right)-\left(\left\lceil\frac{r}{2}\right\rceil+1,\left\lfloor\frac{k}{2}\right\rfloor+1\right)-b_{\left\lfloor\frac{k}{2}\right\rfloor+1},
\end{gathered}
$$

and hence

$$
\begin{gathered}
a_{2}-(1, k)-\left(\left\lceil\frac{r}{2}\right\rceil, k\right)-b_{1}, \\
a_{2}-(1, k-1)-\left(\left\lceil\frac{r}{2}\right\rceil, k-1\right)-b_{k}, \\
\vdots \\
a_{2}-\left(1,\left\lfloor\frac{k}{2}\right\rfloor+2\right)-\left(\left\lceil\frac{r}{2}\right\rceil,\left\lfloor\frac{k}{2}\right\rfloor+2\right)-b_{\left\lfloor\frac{k}{2}\right\rfloor+2} .
\end{gathered}
$$

those geodesics cover $k-1$ layers of $P_{\left\lceil\frac{r}{2}\right\rceil}$. By symmetry we construct other $a_{i}-b_{j}$ geodesics in the same way. Thus,

$$
V\left(\widetilde{I}\left(S_{1}\right)\right)=V\left(P_{\left\lceil\frac{r}{2}\right\rceil} \square C_{n}\right) .
$$

Similarly, the subgraph induced by

$$
V\left(C_{n}^{\left[\frac{r}{2}\right\rceil}\right) \cup V\left(C_{n}^{\left[\frac{r}{2}\right\rceil+1}\right) \cup \ldots \cup V\left(C_{n}^{r}\right),
$$

is isomorphic to $P_{\left\lfloor\frac{r}{2}\right\rfloor+1}^{\prime} \square C_{n}$, where $P_{\left\lfloor\frac{r}{2}\right\rfloor+1}^{\prime} \cong P_{\left\lceil\frac{r}{2}\right\rceil}$ or $P_{\left\lceil\frac{r}{2}\right\rceil+1}$.

Let $S_{2}=\left\{b_{1}, b_{2}, \ldots, b_{k}\right\} \cup\left\{c_{1}, c_{2}, \ldots, c_{k}\right\}$. Then

$$
V\left(\widetilde{I}\left(S_{2}\right)\right)=V\left(P_{\left\lfloor\frac{r}{2}\right\rfloor+1}^{\prime} \square C_{n}\right) .
$$

We conclude $V(\widetilde{I}(S))=V\left(C_{r} \square C_{n}\right)$.

Case $2 n=k^{2}+l$, where $1 \leq l \leq k$.

Let $S=\left\{a_{1}, a_{2}, \ldots, a_{k}\right\} \cup\left\{b_{1}, b_{2}, \ldots, b_{k}\right\} \cup\left\{c_{1}, c_{2}, \ldots, c_{k}\right\} \cup\left\{\left(\left\lceil\frac{r}{2}\right\rceil, k^{2}+\left\lceil\frac{l}{2}\right\rceil\right)\right\}$. Clearly, $|S|=3 k+1 \leq\lceil 3 \sqrt{n}\rceil$.

As the case above, the geodesics consisting by $\left\{a_{1}, a_{2}, \ldots, a_{k}\right\} \cup\left\{b_{1}, b_{2}, \ldots, b_{k}\right\} \cup\left\{c_{1}, c_{2}, \ldots, c_{k}\right\}$ have covered all vertices of $C_{r} \square C_{k^{2}}$, and the geodesics $\left(\left\lceil\frac{r}{2}\right\rceil, k^{2}+\left\lceil\frac{l}{2}\right\rceil\right)-a_{i}$ and $\left(\left\lceil\frac{r}{2}\right\rceil, k^{2}+\left\lceil\frac{l}{2}\right\rceil\right)-c_{i}(i=1,2, \cdots, k)$ cover the vertices of $\left(C_{r} \square C_{k^{2}+l}\right) \backslash\left(C_{r} \square C_{k^{2}}\right)$.

Case $3 n=k^{2}+l$, where $k+1 \leq l \leq 2 k$.

By adding two vertices $\left(\left\lceil\frac{r}{2}\right\rceil, k^{2}+\left\lceil\frac{l}{3}\right\rceil\right)$ and $\left(\left\lceil\frac{r}{2}\right\rceil, k^{2}+2\left\lceil\frac{l}{3}\right\rceil\right)$ to $S$ of Case 1 , we have $|S|=3 k+2 \leq\lceil 3 \sqrt{n}\rceil$.

By Case 2, we have covered all vertices of $C_{r} \square C_{k^{2}+k}$, and the geodesics $\left(\left\lceil\frac{r}{2}\right\rceil, k^{2}+\left\lceil\frac{l}{3}\right\rceil\right)-a_{i},\left(\left\lceil\frac{r}{2}\right\rceil, k^{2}+2\left\lceil\frac{l}{3}\right\rceil\right)-a_{i}$ and $\left(\left\lceil\frac{r}{2}\right\rceil, k^{2}+\left\lceil\frac{l}{3}\right\rceil\right)-c_{i},\left(\left\lceil\frac{r}{2}\right\rceil, k^{2}+2\left\lceil\frac{l}{3}\right\rceil\right)-c_{i}(i=1,2, \cdots, k)$ can cover the vertices of $\left(C_{r} \square C_{k^{2}+l}\right) \backslash\left(C_{r} \square C_{k^{2}+k}\right)$.

The proof is completed. 


\section{Cube}

The $n$-dimensional cube $Q_{n}$ is Cartesian product of $n$ copies of the path $P_{2}$. Let $n \geq 1$, the graph $Q_{n}$ has $2^{n}$ vertices, each labeled by an $n$-bit binary string $u_{1} u_{2} \cdots u_{n}$ such that $u_{i} \in\{0,1\}$ for all $i . Q_{1}$ is isomorphic to the complete graph $K_{2}$ where one vertex is labeled by the digit 0 and the other by 1 . For $n \geq 2, Q_{n}$ is defined recursively by using two copies of $(n-1)$-dimensional cubes with edges between them, the first copy denoted by $Q_{n-1}^{0}$ with vertices $u=0 u_{1} u_{2} \cdots u_{n-1}$, another copy is $Q_{n-1}^{1}$ with vertices $v=1 v_{1} v_{2} \cdots v_{n-1} . Q_{n}$ is a $n$-regular bipartite graph and $\operatorname{diam}\left(Q_{n}\right)=n$. A few examples of cube are shown in Figure 2.
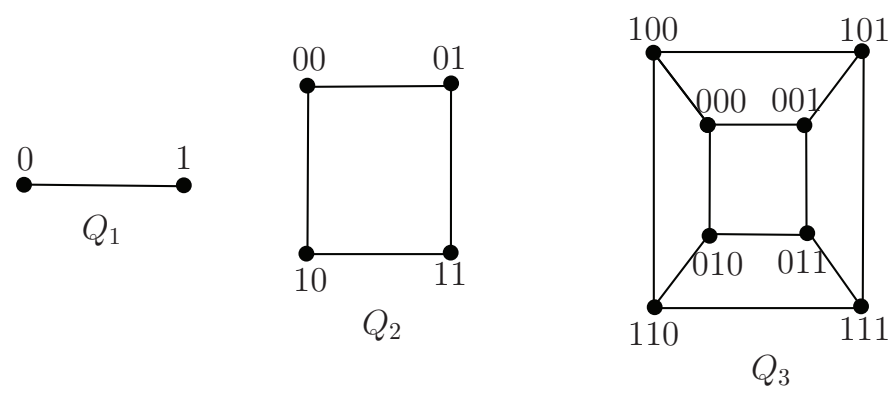

Figure 2. Three cubes

Lemma 3 (1) $\operatorname{sg}\left(Q_{1}\right)=2, \operatorname{sg}\left(Q_{2}\right)=3$.

(2) $\operatorname{sg}\left(Q_{3}\right)=4, \operatorname{sg}\left(Q_{4}\right)=5$.

Proof. (1) The result follows Observation 1 , since $Q_{1} \cong P_{2}$ and $Q_{2} \cong C_{4}$.

(2) Choose $S=\{000,111,010,101\}$, let $\widetilde{I}(S)=\{000-001-011-111,000-100-101,111-110-010\}$. Then $V(\widetilde{I}(S))=V\left(Q_{3}\right)$ and that $S$ is a strong geodetic set. So $\operatorname{sg}\left(Q_{3}\right) \leq 4$.

For any three vertices $\left\{v_{1}, v_{2}, v_{3}\right\}=S \subseteq V\left(Q_{3}\right)$, there are 3 paths $P_{n_{1}}, P_{n_{2}}, P_{n_{3}}$ connecting $\left(v_{1}, v_{2}\right),\left(v_{1}, v_{3}\right),\left(v_{2}, v_{3}\right)$ respectively. There is at most one path with distance 3 . Without loss of generality, let $\left|V\left(P_{n_{1}}\right)\right|=4$ and $d\left(v_{1}, v_{2}\right)=3$. Clearly, $P_{n_{1}}$ covers at most 2 vertices outside of $S$. For $P_{n_{i}}(i=2,3),\left|V\left(P_{n_{i}}\right)\right| \leq 3$ and $d\left(v_{1}, v_{3}\right) \leq 2, d\left(v_{2}, v_{3}\right) \leq 2, P_{n_{i}}(i=2,3)$ covers at most one vertex outside $S$. We conclude that $\bigcup_{i=1}^{3} P_{n_{i}}$ covers at most 4 vertices outside of $S$ and covers at most 7 vertices in $Q_{3}$. From the arbitrariness of $S$, we know that $\operatorname{sg}\left(Q_{3}\right) \geq 4$, and hence $\operatorname{sg}\left(Q_{3}\right)=4$.

For $Q_{4}$, we choose $S=\{0000,1111,0001,1110,1010\}$ and $\widetilde{I}(S)=\{0000-0100-0110-0111-1111,0000-1000-1010-1110$, 1111-1011-1001-0001, 0001-0101-1101-1100-1110, 0001-0011-0010-1010\}. Then $V(\widetilde{I}(S))=V\left(Q_{4}\right)$ and that $S$ is a strong geodetic set. So $s g\left(Q_{4}\right) \leq 5$.

For any four vertices $\left\{v_{1}, v_{2}, v_{3}, v_{4}\right\}=S \subseteq V\left(Q_{4}\right)$, there are 6 geodesics $P_{n_{1}}, P_{n_{2}}, P_{n_{3}}, P_{n_{4}}, P_{n_{5}}, P_{n_{6}}$ connecting $\left(v_{1}, v_{2}\right),\left(v_{1}, v_{3}\right)$, $\left(v_{1}, v_{4}\right),\left(v_{2}, v_{3}\right),\left(v_{2}, v_{4}\right),\left(v_{3}, v_{4}\right)$ respectively. Suppose there are $r$ geodesics with distance 4 . Then for any vertex $v_{i}$ $(1 \leq i \leq 4)$, there is only one vertex $v_{j}(1 \leq j \leq 4, i \neq j)$ such that $d\left(v_{i}, v_{j}\right)=4$, and hence there are at most 2 geodesics of $\left\{P_{n_{1}}, P_{n_{2}}, P_{n_{3}}, P_{n_{4}}, P_{n_{5}}, P_{n_{6}}\right\}$ with distance 4 , that means $r \leq 2$. We consider the following cases by the value of $r$.

Case 1. $r=2$.

Without loss of generality, let $\left|V\left(P_{n_{1}}\right)\right|=\left|V\left(P_{n_{6}}\right)\right|=5$ and $d\left(v_{1}, v_{2}\right)=d\left(v_{3}, v_{4}\right)=4$. Clearly, $P_{n_{i}}(i=1,6)$ covers at most 3 vertices outside of $S$. If there is one geodesic with distance 3 , say $d\left(v_{1}, v_{3}\right)=3$, then $d\left(v_{2}, v_{4}\right)=3, d\left(v_{1}, v_{4}\right)=1$ and $d\left(v_{2}, v_{3}\right)=1$. For example, if $S=\{0000,1111,1110,0001\}$, then $P_{n_{i}}(i=2,5)$ covers at most 2 vertices outside of $S$. We conclude that $\bigcup_{i=1}^{6} P_{n_{i}}$ covers at most 10 vertices outside of $S$ and covers at most 14 vertices in $Q_{4}$. If $d\left(v_{1}, v_{3}\right)=2$, then $d\left(v_{1}, v_{4}\right)=d\left(v_{2}, v_{3}\right)=d\left(v_{2}, v_{4}\right)=2$. If $S=\{0000,1111,1010,0101\}$, then $P_{n_{i}}(i=2,3,4,5)$ covers at most one vertex outside of $S$. We conclude that $\bigcup_{i=1}^{6} P_{n_{i}}$ covers at most 10 vertices outside of $S$ and covers at most 14 vertices in $Q_{4}$. By symmetry of $Q_{4}$, the case $d\left(v_{1}, v_{3}\right)=1$ can be proved similarly as the case $d\left(v_{1}, v_{3}\right)=3$.

Case 2. $r=1$.

Without loss of generality, let $\left|V\left(P_{n_{1}}\right)\right|=5$ and $d\left(v_{1}, v_{2}\right)=4$. Clearly, $\left|V\left(P_{n_{i}}\right)\right| \leq 4(i=2,3, \cdots, 6)$. We claim that there are at most 2 geodesics of $\left\{P_{n_{2}}, P_{n_{3}}, P_{n_{4}}, P_{n_{5}}, P_{n_{6}}\right\}$ with distance 3 . Otherwise, there are 3 geodesics with distance 3 . 
Then there exists one vertex with at least 2 geodesics with distance 3 . If this vertex is $v_{1}$ and $d\left(v_{1}, v_{3}\right)=d\left(v_{1}, v_{4}\right)=3$, then $d\left(v_{3}, v_{4}\right) \leq 2$, and hence $d\left(v_{1}, v_{2}\right) \leq 3$, which contradicting to the fact $d\left(v_{1}, v_{2}\right)=4$. If this vertex is $v_{3}$ and $d\left(v_{1}, v_{3}\right)=d\left(v_{3}, v_{4}\right)=3$, then $d\left(v_{1}, v_{4}\right) \leq 2$, and hence $d\left(v_{1}, v_{2}\right) \leq 3$, also a contradiction. Hence, there are at most 2 geodesics of $\left\{P_{n_{2}}, P_{n_{3}}, \cdots, P_{n_{6}}\right\}$ with distance 3 , say $P_{n_{2}}, P_{n_{3}}$. Clearly, $P_{n_{i}}(i=2,3)$ covers at most 2 vertices outside of $S$ and $d\left(v_{2}, v_{3}\right) \leq 2, d\left(v_{2}, v_{4}\right) \leq 2, d\left(v_{3}, v_{4}\right) \leq 2, P_{n_{i}}(i=4,5,6)$ covers at most one vertex outside of $S$. We conclude that $\bigcup_{i=1}^{6} P_{n_{i}}$ covers at most 10 vertices outside of $S$ and covers at most 14 vertices in $Q_{4}$. If there is only one geodesic, say $P_{n_{2}}$ with distance 3 , then it covers at most 2 vertices outside of $S$, and hence $P_{n_{i}}(3 \leq i \leq 6),\left|V\left(P_{n_{i}}\right)\right| \leq 3$ and $d\left(v_{1}, v_{4}\right) \leq 2$, $d\left(v_{2}, v_{3}\right) \leq 2, d\left(v_{2}, v_{4}\right) \leq 2, d\left(v_{3}, v_{4}\right) \leq 2$. Clearly, $P_{n_{i}}(i=3,4,5,6)$ covers at most one vertex outside of $S, \bigcup_{i=1}^{6} P_{n_{i}}$ covers at most 9 vertices outside of $S$ and covers at most 13 vertices in $Q_{4}$. If there is no geodesic with distance 3 , then $P_{n_{i}}$ $(2 \leq i \leq 6),\left|V\left(P_{n_{i}}\right)\right| \leq 3$ and $d\left(v_{1}, v_{3}\right) \leq 2, d\left(v_{1}, v_{4}\right) \leq 2, d\left(v_{2}, v_{3}\right) \leq 2, d\left(v_{2}, v_{4}\right) \leq 2$ and $d\left(v_{3}, v_{4}\right) \leq 2 . P_{n_{i}}(i=2,3, \cdots, 6)$ covers at most one vertex outside of $S$, and hence $\bigcup_{i=1}^{6} P_{n_{i}}$ covers at most 8 vertices outside of $S$ and covers at most 12 vertices in $Q_{4}$.

Case 3. $r=0$.

We claim that there are at most 3 geodesics of $\left\{P_{n_{1}}, P_{n_{2}}, \cdots, P_{n_{6}}\right\}$ with distance 3 . Otherwise, there are 4 geodesics with distance 3 , it must be one vertex, say $v_{1}$ such that $d\left(v_{1}, v_{2}\right)=d\left(v_{1}, v_{3}\right)=3$. Clearly, $d\left(v_{2}, v_{3}\right) \leq 2$. If $d\left(v_{3}, v_{4}\right)=3$, then $d\left(v_{1}, v_{4}\right) \leq 2$ and $d\left(v_{2}, v_{4}\right) \leq 2$, and hence there is no fourth geodesic with distance 3 . If $d\left(v_{1}, v_{4}\right)=3$, then $d\left(v_{3}, v_{4}\right) \leq 2$ and $d\left(v_{2}, v_{4}\right) \leq 2$, also a contradiction. Without loss of generality, let $d\left(v_{1}, v_{2}\right)=d\left(v_{1}, v_{3}\right)=d\left(v_{1}, v_{4}\right)=3$. Clearly, $P_{m_{i}}$ $(i=1,2,3)$ covers at most 2 vertices outside of $S$ and $d\left(v_{2}, v_{3}\right) \leq 2, d\left(v_{2}, v_{4}\right) \leq 2, d\left(v_{3}, v_{4}\right) \leq 2$, and hence $P_{m_{i}}(i=4,5,6)$ covers at most one vertex outside of $S$. We conclude that $\bigcup_{i=1}^{6} P_{m_{i}}$ covers at most 9 vertices outside of $S$ and covers at most 13 vertices in $Q_{4}$.

From the arbitrariness of $S$, we know that $\operatorname{sg}\left(Q_{4}\right) \geq 5$, and hence $\operatorname{sg}\left(Q_{4}\right)=5$.

Theorem $6 \operatorname{sg}\left(Q_{5}\right)=6$.

Proof. Let $S=\{00000,11111,10101,01010,10111,01000\}$ and $\widetilde{I}(S)=\{00000-100000-10001-11001-11011-11111,00000-$ 00001-00101-10101,00000-00010-01010, 00000-00100-00110-00111-10111, 11111-11101-10101, 11111-01111-0101101010, 11111-11110-11100-11000,10101-10100-10110-10010-11010-01010, 10101-11101-01101-01001-01000, 0101000010-00011-10011-10111, 10111-11111-11110-01110-01100-01000\}, then $V(\widetilde{I}(S))=V\left(Q_{5}\right)$ and $\operatorname{sg}\left(Q_{5}\right) \leq 6$.

For any five vertices $\left\{v_{1}, v_{2}, v_{3}, v_{4}, v_{5}\right\}=S \subseteq V\left(Q_{5}\right)$, there are 10 geodesics $P_{n_{1}}, P_{n_{2}}, P_{n_{3}}, P_{n_{4}}, P_{n_{5}}, P_{n_{6}}, P_{n_{7}}, P_{n_{8}}, P_{n_{9}}, P_{n_{10}}$ connecting $\left(v_{1}, v_{2}\right),\left(v_{1}, v_{3}\right),\left(v_{1}, v_{4}\right),\left(v_{1}, v_{5}\right),\left(v_{2}, v_{3}\right),\left(v_{2}, v_{4}\right),\left(v_{2}, v_{5}\right),\left(v_{3}, v_{4}\right),\left(v_{3}, v_{5}\right),\left(v_{4}, v_{5}\right)$ respectively. Suppose there are $r$ geodesics with distance 5 , for any vertex $v_{i}(1 \leq i \leq 5)$, there must exist only one vertex $v_{j}(1 \leq j \leq 5, i \neq j)$ such that $d\left(v_{i}, v_{j}\right)=5$ and there are at most 2 geodesics of $P_{n_{i}}(i=1,2, \cdots, 10)$ with distance 5 , that means $r \leq 2$. We consider the following cases by the value of $r$.

Case 1. $r=2$.

Without loss of generality, let $\left|V\left(P_{n_{1}}\right)\right|=\left|V\left(P_{n_{8}}\right)\right|=6$ and $d\left(v_{1}, v_{2}\right)=d\left(v_{3}, v_{4}\right)=5$. Clearly $P_{n_{i}}(i=1,8)$ covers at most 4 vertices outside of $S$. Suppose there is one geodesic of $\left\{P_{n_{2}}, \cdots, P_{n_{7}}, P_{n_{9}}, P_{n_{10}}\right\}$ with distance 4 , say $d\left(v_{1}, v_{3}\right)=4$, then $d\left(v_{2}, v_{4}\right)=4, d\left(v_{1}, v_{4}\right)=1$ and $d\left(v_{2}, v_{3}\right)=1$. If the distance between $v_{5}$ and one of $v_{i}(1 \leq i \leq 4)$ is 4 , say $d\left(v_{5}, v_{1}\right)=4$, then $d\left(v_{5}, v_{2}\right)=1, d\left(v_{5}, v_{3}\right)=3, d\left(v_{5}, v_{4}\right)=2$. And hence there are at most 3 geodesics of $P_{n_{i}}(i \neq 1,8)$ with distance 4 . Clearly, $P_{n_{i}}(i=2,4,6)$ covers at most 3 vertices outsides of $S, P_{n_{9}}$ covers at most 2 vertices outside of $S, P_{n_{10}}$ covers at most one vertex outside of $S$. We conclude that $\bigcup_{i=1}^{10} P_{n_{i}}$ covers at most 20 vertices outside of $S$ and covers at most 25 vertices in $Q_{5}$. If there are two geodesics with distance 4, say $d\left(v_{5}, v_{1}\right)=d\left(v_{5}, v_{3}\right)=4$, then $P_{n_{i}}(i=4,9)$ covers at most 3 vertices outside of $S, P_{n_{i}}(i=2,3,5,6,7,10)$ covers at most 2 vertices outside of $S . \bigcup_{i=1}^{10} P_{n_{i}}$ covers at most 26 vertices outside of $S$ and covers at most 31 vertices in $Q_{5}$. If there is only one geodesic with distance 4, say $d\left(v_{5}, v_{1}\right)=4$, then $P_{n_{4}}$ covers at most 3 vertices outside of $S, P_{n_{i}}(i=2,3,5,6,7,9,10)$ covers at most 2 vertices outside of $S$. $\bigcup_{i=1}^{10} P_{n_{i}}$ covers at most 25 vertices outside of $S$ and covers at most 30 vertices in $Q_{5}$. If there is no geodesic with distance $4, P_{n_{i}}(1 \leq i \leq 10$, $i \neq 1,8)$ covers at most 2 vertices outside of $S$, then $\bigcup_{i=1}^{10} P_{n_{i}}$ covers at most 24 vertices outside of $S$ and covers at most 29 vertices in $Q_{5}$.

Case 2. $r=1$.

Let $\left|V\left(P_{n_{1}}\right)\right|=6$ and $d\left(v_{1}, v_{2}\right)=5, P_{n_{1}}$ covers at most 4 vertices outside of $S$. First we claim that there are at least 3 geodesics with distance 4 of $\left\{P_{n_{2}}, P_{n_{3}}, \cdots, P_{n_{10}}\right\}$. Otherwise there are 2 geodesics with distance 4 , say $P_{n_{2}}, P_{n_{3}}$ and $P_{n_{i}}$ $(i=2,3)$ covers at most 3 vertices outside of $S$. Clearly, $P_{n_{i}}(i=4,5, \cdots, 10)$ covers at most 2 vertices outside of $S$, we conclude $\bigcup_{i=1}^{10} P_{n_{i}}$ covers at most 24 vertices outside of $S$ and covers at most 29 vertices in $Q_{5}$, a contradiction. For any vertex $v_{i}(3 \leq i \leq 5)$, there is at most one geodesic with distance 4 of the two geodesics connecting $v_{i}$ and $v_{j}(j=1,2)$, at most 3 geodesics with distance 4 of the set of geodesics $\left\{P_{n_{2}}, P_{n_{3}}, P_{n_{4}}, P_{n_{5}}, P_{n_{6}}, P_{n_{7}}\right\}$ connecting $v_{i}(3 \leq i \leq 5)$ and $v_{j}$ 
$(j=1,2)$. Let $t$ be the number of geodesics with distance 4 connecting $v_{i}(3 \leq i \leq 5)$ and $v_{j}(j=1,2)$, we consider the subcases as follows by the value of $t$.

Subcase 1. $t=3$.

Suppose $d\left(v_{1}, v_{3}\right)=d\left(v_{1}, v_{4}\right)=d\left(v_{1}, v_{5}\right)=4$, then $d\left(v_{3}, v_{4}\right)=d\left(v_{3}, v_{5}\right)=d\left(v_{4}, v_{5}\right)=2, d\left(v_{2}, v_{3}\right)=d\left(v_{2}, v_{4}\right)=d\left(v_{2}, v_{5}\right)=$ 1. Clearly, $P_{n_{i}}(i=2,3,4)$ covers at most 3 vertices outside of $S, P_{n_{i}}(i=8,9,10)$ covers at most one vertex outside of $S, \bigcup_{i=1}^{10} P_{n_{i}}$ covers at most 16 vertices outside of $S$ and covers at most 21 vertices in $Q_{5}$. Suppose $d\left(v_{1}, v_{4}\right)=d\left(v_{1}, v_{5}\right)=$ $d\left(v_{2}, v_{3}\right)=4$, then $d\left(v_{3}, v_{4}\right)=d\left(v_{3}, v_{5}\right)=3, d\left(v_{4}, v_{5}\right)=2, d\left(v_{1}, v_{3}\right)=d\left(v_{2}, v_{4}\right)=d\left(v_{2}, v_{5}\right)=1 . P_{n_{i}}(i=3,4,5)$ covers at most 3 vertices outside of $S, P_{n_{i}}(i=8,9)$ covers at most 2 vertices outside of $S, P_{n_{10}}$ covers at most one vertex outside of $S, \bigcup_{i=1}^{10} P_{n_{i}}$ covers at most 18 vertices outside of $S$ and covers at most 23 vertices in $Q_{5}$.

Subcase 2. $t=2$.

Suppose $d\left(v_{1}, v_{4}\right)=d\left(v_{1}, v_{5}\right)=4$. If $d\left(v_{3}, v_{5}\right)=4$, then $d\left(v_{3}, v_{4}\right)=4$ or $d\left(v_{3}, v_{4}\right)=2$. If $d\left(v_{3}, v_{4}\right)=2$, then $d\left(v_{3}, v_{4}\right)=4$, $d\left(v_{2}, v_{3}\right)=3, d\left(v_{1}, v_{3}\right)=d\left(v_{4}, v_{5}\right)=2, d\left(v_{2}, v_{5}\right)=1 . P_{n_{i}}(i=3,4,9)$ covers at most 3 vertices outside of $S, P_{n_{5}}$ covers at most 2 vertices outside of $S, P_{n_{i}}(i=2,8,10)$ covers at most one vertex outside of $S, \bigcup_{i=1}^{10} P_{n_{i}}$ covers at most 18 vertices outside of $S$ and covers at most 23 vertices in $Q_{5}$. If $d\left(v_{3}, v_{4}\right)=4$, then $d\left(v_{3}, v_{4}\right)=4, d\left(v_{2}, v_{3}\right)=3$, $d\left(v_{1}, v_{3}\right)=d\left(v_{4}, v_{5}\right)=2, d\left(v_{2}, v_{5}\right)=1, P_{n_{i}}(i=3,4,8,9)$ covers at most 3 vertices outside of $S, P_{n_{5}}$ cover at most 2 vertices outside of $S, P_{n_{i}}(i=2,10)$ covers at most one vertex outside of $S, \bigcup_{i=1}^{10} P_{n_{i}}$ covers at most 20 vertices outside of $S$ and covers at most 25 vertices in $Q_{5}$. Suppose $d\left(v_{1}, v_{5}\right)=d\left(v_{2}, v_{3}\right)=4$. If $d\left(v_{3}, v_{4}\right)=4$, then $d\left(v_{3}, v_{5}\right)=3$ or $d\left(v_{3}, v_{5}\right)=5$. If $d\left(v_{3}, v_{5}\right)=5$, then there are 2 geodesics with distance 5, a contradiction. If $d\left(v_{3}, v_{5}\right)=3$, then $d\left(v_{1}, v_{4}\right)=d\left(v_{4}, v_{5}\right)=3$, $d\left(v_{2}, v_{4}\right)=2, d\left(v_{1}, v_{3}\right)=d\left(v_{2}, v_{5}\right)=1 . P_{n_{i}}(i=4,5,8)$ covers at most 3 vertices outside of $S, P_{n_{i}}(i=3,9,10)$ covers at most 2 vertices outside of $S, P_{n_{6}}$ covers at most one vertex outside of $S, \bigcup_{i=1}^{10} P_{n_{i}}$ covers at most 20 vertices outside of $S$ and covers at most 25 vertices in $Q_{5}$.

Subcase 3. $t=1$.

Suppose $d\left(v_{1}, v_{5}\right)=4$ and $d\left(v_{4}, v_{5}\right)=4$. If $d\left(v_{3}, v_{4}\right)=4$, then $d\left(v_{1}, v_{3}\right)=4, d\left(v_{2}, v_{4}\right)=3, d\left(v_{1}, v_{4}\right)=d\left(v_{3}, v_{5}\right)=2$, $d\left(v_{2}, v_{3}\right)=d\left(v_{2}, v_{5}\right)=1$. Clearly, $P_{n_{i}}(i=2,4,8,10)$ covers at most 3 vertices outside of $S, P_{n_{6}}$ covers at most 2 vertices outside of $S, P_{n_{i}}(i=3,9)$ covers at most 1 vertex outside of $S, \bigcup_{i=1}^{10} P_{n_{i}}$ covers at most 20 vertices outside of $S$ and covers at most 25 vertices in $Q_{5}$. If $d\left(v_{3}, v_{5}\right)=4$, then $d\left(v_{2}, v_{3}\right)=d\left(v_{2}, v_{4}\right)=3, d\left(v_{1}, v_{3}\right)=d\left(v_{1}, v_{4}\right)=d\left(v_{3}, v_{4}\right)=2, d\left(v_{2}, v_{5}\right)=1$. $P_{n_{i}}(i=4,9,10)$ covers at most 3 vertices outside of $S, P_{n_{i}}(i=5,6)$ covers at most 2 vertices outside of $S, P_{n_{i}}(i=2,3,8)$ covers at most one vertex outside of $S, \bigcup_{i=1}^{10} P_{n_{i}}$ covers at most 20 vertices outside of $S$ and covers at most 25 vertices in $Q_{5}$.

Subcase 4. $t=0$.

Clearly, $d\left(v_{3}, v_{4}\right)=d\left(v_{3}, v_{5}\right)=d\left(v_{4}, v_{5}\right)=4$. Let $v_{3}=00000, v_{4}=01111$. Then $v_{5}$ must be one in $\{10111,11011,11101$, $11110\}$, and hence $d\left(v_{4}, v_{5}\right)=2$, a contradiction.

Case 3. $r=0$.

From the previous proof, there are at most 5 geodesics with distance 4 of the set of geodesics $\left\{P_{n_{1}}, P_{n_{2}}, \cdots, P_{n_{10}}\right\}$. If $d\left(v_{1}, v_{2}\right)=d\left(v_{2}, v_{3}\right)=4$, then $d\left(v_{3}, v_{4}\right)=4, d\left(v_{4}, v_{5}\right)=4, d\left(v_{5}, v_{1}\right)=4$, we have $d\left(v_{1}, v_{3}\right)=d\left(v_{1}, v_{4}\right)=d\left(v_{2}, v_{4}\right)=$ $d\left(v_{2}, v_{5}\right)=d\left(v_{3}, v_{5}\right)=2$, then $P_{n_{i}}(i=1,4,5,8,10)$ covers at most 3 vertices outside of $S, P_{n_{i}}(i=2,3,6,7,9)$ covers at most one vertex outside of $S$, we conclude that $\bigcup_{i=1}^{10} P_{n_{i}}$ covers at most 20 vertices outside of $S$ and cover at most 25 vertices in $Q_{5}$. For example, $S=\{00000,11110,00011,10100,01111\}$. If there are less than 5 geodesics with distance 4 , then $\bigcup_{i=1}^{10} P_{n_{i}}$ can not cover 32 vertices of $Q_{5}$.

From the arbitrariness of $S$, we have $s g\left(Q_{5}\right) \geq 6$, and hence $\operatorname{sg}\left(Q_{5}\right)=6$.

The following example shows that for $Q_{2}$, the equality holds for Theorem 1. Clearly,

$$
\begin{aligned}
\operatorname{sg}\left(Q_{2}\right)=3 & =\operatorname{sg}\left(Q_{1} \square P_{2}\right) \\
& =\min \left\{V\left(Q_{1}\right)\left(\operatorname{sg}\left(P_{2}\right)-1\right)+1, V\left(P_{2}\right)\left(\operatorname{sg}\left(Q_{1}\right)-1\right)+1\right\} .
\end{aligned}
$$

For $\operatorname{sg}\left(Q_{n}\right)(n=8)$, if there exists a geodetic set $S$ of cardinality $n+1$ satisfying $V(\widetilde{I}(S))=V\left(Q_{n}\right)$. In fact, there are exactly 36 geodesics. But for any vertex $u \in S$, there is only one vertex $v \in S$ such that $d(u, v)=8$, and hence we can find at most 4 geodesics with distance 8 . It is clear that the remaining 32 geodesics can not cover other vertices. So, we get an upper bound as follow:

Theorem 7 For $Q_{n}(n \geq 5), \operatorname{sg}\left(Q_{n}\right) \leq 2^{n-5} \times 5+1$. 
Proof. From Theorem 6, we have

$$
\begin{aligned}
\operatorname{sg}\left(Q_{n}\right) & =\operatorname{sg}\left(Q_{n-1} \square P_{2}\right) \\
& \leq \min \left\{V\left(Q_{n-1}\right)\left(\operatorname{sg}\left(P_{2}\right)-1\right)+1, V\left(P_{2}\right)\left(\operatorname{sg}\left(Q_{n-1}\right)-1\right)+1\right\} \\
& =\min \left\{2^{n-1}+1,2 \operatorname{sg}\left(Q_{n-1}\right)-1\right\}
\end{aligned}
$$

when $n \geq 5$. Then $2^{n-1}+1 \geq 2 \operatorname{sg}\left(Q_{n-1}\right)-1$, and hence

$$
\begin{aligned}
\operatorname{sg}\left(Q_{n}\right) & \leq 2\left(\operatorname{sg}\left(Q_{n-1}\right)-1\right)+1 \\
& \leq 2^{2}\left(\operatorname{sg}\left(Q_{n-2}\right)-1\right)+1 \\
& \leq 2^{3}\left(\operatorname{sg}\left(Q_{n-3}\right)-1\right)+1 \\
& \vdots \\
& \leq 2^{n-5}\left(\operatorname{sg}\left(Q_{5}\right)-1\right)+1 \\
& =2^{n-5} \times 5+1 .
\end{aligned}
$$

\section{Further Research}

In this paper, we have studied the strong geodesic problem on the torus network and some other Cartesian product networks. Next we can try to determine the exact strong geodetic number for $Q_{n}(n \geq 6)$ and consider the strong geodetic number on Lexicographic product, Strong product and Direct product.

\section{References}

Atici, M. (2002). Computational complexity of geodetic set, Int. J. Comput. Math. 79, 587-591. https://doi.org/10.1080/00207160210954

Bao, F., Igarashi, Y., \& Öhring, S. R. (1998). Reliable Broadcasting in Product Networks, Discrete Applied Math, 83, 3-20. https://doi.org/10.1016/S0166-218X(97)00100-5

Bondy, J. A., \& Murty, U. S. R. (2008). Graph theory, GTM 244, Springer.

Brešar, B., Kovše, M., \& Tepeh, A. (2011). Geodetic sets in graphs, in: M. Dehemr(Ed). Structural Analysis of Complex Networks, Birkhäuser, Boston, 197-218.

Brešar, B., \& Tepeh, H. A. (2008). On the geodetic number of median graphs, Discrete Math, 308, 4044-4051.

Buckley, F., Harary, F., \& Quintas, L. V. (1998). Extremal results on the geodetic number of a graph, Scientia 2A, 17-26.

Chartrand, G., Harary, F., \& Zhang, P. (2000). Geodetic sets in graph, Discuss. Math. Graph Theory, 20, 129-138. https://doi.org/10.7151/dmgt.1112

Chartrand, G., Harary, F., \& Zhang, P. (2002). On the geodetic number of a graph, Networks, 39 , 1-6. https://doi.org/10.1002/net.10007

Chartrand, G., Palmer, E. M., \& Zhang, P. (2002). The geodetic number of a graph: A survey, Congr. Numer, 156, 37-58.

Das, S. K., Öhring, S. R., \& Banerjee, A. K. (1995). Embeddings into hyper Petersen network: Yet another hypercube-like interconnection topology, VLSI Design, 2(4), 335-351. https://doi.org/10.1155/1995/95759

Day, K., \& AL-Ayyoub, A. E. (1997). The cross product of interconnection networks, IEEE Trans. Parall. Distr. Sys, $8(2), 109-118$.

Fitzpatrick, S. L. (1999). The isometric path number of the Cartesian product of paths, Congr. Numer, 137, 109-119.

Hammack, R., Imrich, W., \& Klavžar, S. (2001). Handbook of product graphs, Second edition, CRC Press.

Harary, F., Loukakis, E., \& Tsouros, C. (1993). The geodetic number of a graph, Math. Comput. Modelling, 17, 89-95. https://doi.org/10.1016/0895-7177(93)90259-2

Ir ̌̌s $\breve{c}$, V. (2018). Strong geodetic numbers of complete bipartite graphs and of graphs with specified diameter, Graphs and Combinatorics, 34(3), 443-456.

Klavžar, S., \& Manuel, P. (2018). Strong geodetic problem in grid like architectures, Bulletion of the Malaysian Mathematical Sciences Society. 41(3), 1671-1680. 
Ku, S., Wang, B., \& Hung, T. (2003). Constructing edge-disjoint spanning trees in product networks, IEEE Trans. Parall. Distr. Sys. 14(3), 213-221.

Manuel, P., Klavžar, S., Xavier, A., Arokiaraj, A., \& Thomas, E. (2018). Strong edge geodetic problem in networks, Discuss. Math. Graph Theory. https://doi.org/10.7151/dmgt.2139

Manuel, P., Klavžar, S., Xavier, A., Arokiaraj, A., \& Thomas, E. (2017). Strong geodetic problem in networks: computational complexity and solution for Apollonian networks.

Ye, Y., Lu, C., \& Liu, Q. (2007). The geodetic numbers of Cartesian products of graphs, Math. Appl. (Wuhan), 20, $158-163$.

\section{Copyrights}

Copyright for this article is retained by the author(s), with first publication rights granted to the journal.

This is an open-access article distributed under the terms and conditions of the Creative Commons Attribution license (http://creativecommons.org/licenses/by/4.0/). 